\title{
Improvement of diabetic control by continuous subcutaneous insulin infusion therapy changes fatty acid composition of serum lipids and erythrocytes in Type 1 (insulin-dependent) diabetes
}

\author{
R. S. Tilvis, E. Helve and T.A. Miettinen \\ Second and Third Departments of Medicine, University of Helsinki, Helsinki, Finland
}

\begin{abstract}
Summary. The influence of improved diabetic control on the fatty composition of serum lipids, erythrocytes and platelets was investigated in 24 patients with Type 1 (insulin-dependent) diabetes treated for 6 months with either continuous subcutaneous insulin infusion $(n=14)$ or conventional insulin therapy $(n=10)$. The groups were matched for age, sex, body mass index, serum lipids, duration of diabetes, glycosylated haemoglobin and insulin dose. Glycaemic control improved, and the contents of dihomogammalinolenic acid and arachidonic acid but not linoleic acid rose significantly $(p<0.05)$, in serum lipids of patients treated with continuous infusion. No changes were observed in the group treated with insulin injec-
\end{abstract}

tions. Both in serum and erythrocytes the n- 6 polyunsaturated fatty acid ratios rose consistently in the patients, with improvement of control regardless of the mode of treatment. Furthermore, the change of $\mathrm{HbA}_{1}$ was negatively correlated with that of arachidonic acid in erythrocytes. No changes were found in the platelet fatty acid compositions. The findings suggest that improved diabetic control enhances the conversion of linoleic acid to arachidonic acid, probably by activating enzymes needed for chain elongation and desaturation.

Key words: Diabetes mellitus, fatty acids, serum lipids, erythrocytes, platelets.
Human diabetes mellitus is often associated with disturbances of lipid metabolism. As compared to serum triglyceride and cholesterol levels and lipoprotein metabolism, relatively little attention has been paid to the fatty acid composition of serum lipids and blood cell membranes in human Type 1 (insulin-dependent) diabetes. However, both human studies [1-3] and animal experiments [4-10] have indicated that the insulin deficit may affect the metabolism of essential fatty acids. The conversion of essential fatty acids like linoleic acid $(18: 2, \mathrm{n}-6)$ to longer-chain polyunsaturated fatty acids (PUFA), e.g. dihomogammalinolenic acid (DHGLA, 20:3,n-6) and arachidonic acid (ARA 20:4,n-6), is of particular interest because of their role as prostanoid precursors [11]. In fact, several abnormalities of the platelet function and prostaglandin metabolism have been described in human diabetes [12-15]. Cross-sectional studies have shown that ARA-content of diabetic platelets may be increased [17, 18], normal [19] or decreased [20], the latter especially in cases with severe retinopathy [20]. We have recently observed that in women with Type 1 diabetes the content of linoleic acid of serum lipids was higher and that of ARA and other n-6 PUFA lower than in controls [3]. Furthermore, glycosylated haemoglobin $\left(\mathrm{HbA}_{1}\right)$ was positively correlated with the linoleic acid content but, if anything, negatively with the proposed n-6 PUFA metabolites of linoleic acid, suggesting that altered linoleic acid metabolism was related to diabetic control. The present study was designed to explore whether improved diabetic control with continuous subcutaneous insulin infusion therapy (CSII) affects the fatty acid composition of serum and cell membrane lipids in Type 1 diabetes.

\section{Subjects and methods}

\section{Subjects}

Twenty-four patients with Type 1 diabetes were selected from the outpatient diabetes clinic of Helsinki University Central Hospital (Table 1). The patients were participants of the WHO Multicenter Study on the feasibility of continuous subcutaneous insulin infusion therapy (CSII). Ten patients were randomized to continue conventional insulin injection therapy (CIT) for 6 months, whereas 14 patients started CSII for 6 months. The two groups were matched for sex, age, serum lipids, duration and severity of diabetes, glycosylated haemoglobin $\left(\mathrm{HbA}_{1}\right)$ and daily insulin dose.

CSII was performed using the Nordisk Insulin Infuser-pump (Nordisk Insulin Laboratories, Gentofte, Denmark). Approximately $50 \%$ of the daily dose of insulin was given as a continuous infusion and the rest as boluses before meals. CIT consisted of two or three 
daily injections. Velosulin $100 \mathrm{U}$ (Nordisk Insulin Laboratories, Gentofte, Denmark) was used in CSII, and a mixture of intermediate-acting and short-acting insulins (Lente and Actrapid; Novo A/S, Copenhagen, Denmark) in CIT. The diet was controlled before and during the treatment period and showed no detectable change. The energy intake $\left(30 \mathrm{kcal} \cdot \mathrm{kg}^{-1} \cdot \mathrm{day}^{-1}\right)$ contained $45 \%$ carbohydrates, $20 \%$ protein and 35\% fat, the nature of which was kept constant.

Table 1. Clinical data of 24 Type 1 diabetic patients

\begin{tabular}{lcc}
\hline Variable & Treatment \\
\cline { 2 - 3 } & CSII & CIT \\
\hline Number (m/f) & $4 / 10$ & $5 / 5$ \\
Age (years) & $31 \pm 7$ & $35 \pm 10$ \\
BMI & $23.6 \pm 2.7$ & $23.4 \pm 1.9$ \\
Diabetes & & \\
$\quad$ Duration (years) & $14 \pm 8$ & $11 \pm 6$ \\
$\quad$ Complications: & & \\
$\quad$ Retinopathy & 6 & 3 \\
$\quad$ Neuropathy & 2 & 2 \\
$\quad$ Nephropathy & 2 & 0 \\
Treatment time (months) & $5.8 \pm 1.5$ & $6.0 \pm 0.3$ \\
HbA (\%) & & \\
$\quad$ Before & $10.8 \pm 1.5$ & $10.0 \pm 1.3$ \\
$\quad$ After & $9.2 \pm 1.1^{\mathrm{a}}$ & $10.4 \pm 1.6$ \\
Insulin dose (U/day) & & \\
$\quad$ Before & & \\
$\quad$ After & $45 \pm 10$ & $44 \pm 10$ \\
Fasting blood glucose (mmol/l) & & \\
$\quad$ Before & $11.1 \pm 4.9$ & $7.1 \pm 5.2$ \\
$\quad$ After & $11.7 \pm 6.0$ & $10.1 \pm 6.0$ \\
Serum lipids (mmol/l) & & \\
$\quad$ Cholesterol & & \\
$\quad$ Before & & \\
$\quad$ After & & \\
$\quad$ Triglycerides & & \\
$\quad$ After & & \\
\hline
\end{tabular}

Values are the mean \pm SD. CSII $=$ continuous subcutaneous insulin infusion, $\mathrm{CIT}=$ conventional injection therapy.

${ }^{a} p<0.05$

\section{Methods}

Following an overnight fast, blood samples were drawn into two $10-\mathrm{ml}$ tubes and erythrocytes were centrifuged at $125 \mathrm{~g}$ and washed twice with $0.15 \mathrm{mmol} / 1$ saline. Serum cholesterol and triglycerides were measured by standard hospital laboratory methods [21, 22]. A portion of serum was centrifuged at $250 \mathrm{~g}$ for $15 \mathrm{~min}$ to prepare platelet-rich plasma. Platelet-rich plasma was then recentrifuged $(1950 \mathrm{~g})$ and the platelet pellet was washed twice with $0.15 \mathrm{mmol} / 1$ saline. Erythrocytes and platelets were frozen immediatedly.

Glycosylated haemoglobin $A_{1}$ was measured by the microcolumn method [23] after overnight dialysis of haemolysate in saline [24]. The normal values of $\mathrm{HbA}_{1}$ range from $6.0 \%$ to $9.0 \%$.

Serum lipids were extracted with chloroform-methanol $(2: 1, \mathrm{v} / \mathrm{v})$. Triglycerides, cholesterol esters and phospholipids were separated on plastic silica gel chromatoplates using heptane: ethyl ether: acetic acid $(80: 20: 2 \mathrm{~V} / \mathrm{v} / \mathrm{v})$ as a solvent. After addition of trimargarin as an internal standard isolated serum lipids, erythrocytes and platelets were saponified with $1 \mathrm{~N} \mathrm{NaOH}$ in $90 \%$ ethanol for $1 \mathrm{~h}$ at $80^{\circ} \mathrm{C}$. After removal of nonsaponitiable lipids and acidification of the saponification mixture, free fatty acids were extracted with n-heptane and methylated with $5 \%$ methanol-HCl for $2 \mathrm{~h}$ at $85^{\circ} \mathrm{C}$.

The fatty acids were analyzed with a Varian 2100 gas chromatograph (Varian Associates, Palo Alto, Calif., USA) equipped with a 35-mm glass capillary BDS (butane-1,4-diol succinate) column. The temperature programme was $2{ }^{\circ} \mathrm{C} / \mathrm{min}$, from $130^{\circ} \mathrm{C}$ to $200^{\circ} \mathrm{C}$. The peaks were identified on the basis of retention times recorded for different standards and measured with an electronic integrator.

\section{Statistical analysis}

The differences of the fatty acid composition between the groups were tested with an analysis of variance (Anova). The changes of individual fatty acids were tested with a paired two-tailed Student's t-test.

\section{Results}

CSII significantly reduced $\mathrm{HbA}_{1}(p<0.05)$ and insulin requirement, whereas no changes were found in the CIT group (Table 1). Prior to the treatment period, the content and composition of fatty acids of serum lipids, erythrocytes and platelets were virtually similar in the two groups (Table 2).

Table 2. Fatty acid compositions of serum lipids in Type 1 diabetic patients before and after treatment with continuous subcutaneous insulin infusion (CSII) and conventional injection therapy (CIT)

\begin{tabular}{|c|c|c|c|c|c|c|c|c|c|c|c|c|}
\hline \multirow[t]{3}{*}{ Fatty acid ${ }^{a}$} & \multicolumn{4}{|c|}{ Triglycerides } & \multicolumn{4}{|c|}{ Cholesterol esters } & \multicolumn{4}{|c|}{ Phospholipids } \\
\hline & \multicolumn{2}{|l|}{ CSII } & \multicolumn{2}{|l|}{ CIT } & \multicolumn{2}{|l|}{ CSII } & \multicolumn{2}{|l|}{ CIT } & \multicolumn{2}{|l|}{$\overline{\mathrm{CSII}}$} & \multicolumn{2}{|l|}{ CIT } \\
\hline & Before & After & Before & After & Before & After & Before & After & Before & After & Before & After \\
\hline 14:0 & $15 \pm 1$ & $18 \pm 2$ & $15 \pm 2$ & $16 \pm 2$ & $9 \pm 1$ & $8 \pm 1$ & $7 \pm 1$ & $7 \pm 1$ & $3 \pm 0$ & $3 \pm 0$ & $2 \pm 0$ & $3 \pm 0$ \\
\hline $16: 0$ & $268 \pm 6$ & $261 \pm 7$ & $256 \pm 11$ & $252 \pm 9$ & $111 \pm 2$ & $107 \pm 2$ & $105 \pm 1$ & $101 \pm 2$ & $288 \pm 5$ & $285 \pm 6$ & $286 \pm 7$ & $279 \pm 4$ \\
\hline $16: 1, n-7$ & $41 \pm 3$ & $44 \pm 2$ & $39 \pm 3$ & $41 \pm 4$ & $38 \pm 2$ & $36 \pm 3$ & $29 \pm 3^{b}$ & $30 \pm 3$ & $5 \pm 1$ & $6 \pm 1$ & $5 \pm 1$ & $6 \pm 1^{c}$ \\
\hline $18: 0$ & $52 \pm 3$ & $50 \pm 4$ & $50 \pm 3$ & $49 \pm 6$ & $11 \pm 1$ & $10 \pm 1$ & $10 \pm 1$ & $10 \pm 1$ & $137 \pm 2$ & $138 \pm 4$ & $132 \pm 3$ & $132 \pm 3$ \\
\hline $18: 1, n-9$ & $422 \pm 10$ & $422 \pm 7$ & $401 \pm 14$ & $417 \pm 10$ & $206 \pm 7$ & $207 \pm 6$ & $188 \pm 8$ & $189 \pm 10$ & $136 \pm 3$ & $135 \pm 3$ & $126 \pm 4$ & $129 \pm 5$ \\
\hline $18: 2, \mathrm{n}-6$ & $146 \pm 12$ & $148+13$ & $189 \pm 20$ & $173 \pm 17$ & $563 \pm 13$ & $559 \pm 10$ & $594 \pm 12$ & $595 \pm 10^{\mathrm{b}}$ & $245 \pm 11$ & $235 \pm 6$ & $262 \pm 8$ & $265 \pm 9^{b}$ \\
\hline $18: 3, \mathrm{n}-3$ & $10 \pm 1$ & $11 \pm 1$ & $11 \pm 1$ & $13 \pm 2$ & $8 \pm 3$ & $8 \pm 1$ & $8 \pm 3$ & $7 \pm 1$ & $3 \pm 0$ & $3 \pm 0$ & $2 \pm 0$ & $3 \pm 0$ \\
\hline $20: 3, n-9$ & $8 \pm 2$ & $8 \pm 2$ & $6 \pm 1$ & $5 \pm 1$ & $1 \pm 0$ & $1 \pm 0$ & $1 \pm 0$ & $1 \pm 0$ & $5 \pm 0$ & $5 \pm 0$ & $5 \pm 0$ & $5 \pm 0$ \\
\hline $20: 3, \mathrm{n}-6$ & $1 \pm 0$ & $2 \pm 0^{\circ}$ & $2 \pm 0$ & $2 \pm 0$ & $3 \pm 0$ & $4 \pm 0^{c}$ & $4 \pm 0$ & $4 \pm 0$ & $16 \pm 1$ & $19 \pm 1^{c}$ & $17 \pm 2$ & $17 \pm 2$ \\
\hline $20: 4, n-6$ & $9 \pm 1$ & $10 \pm 1^{\mathrm{c}}$ & $10 \pm 1$ & $10 \pm 1$ & $36 \pm 2$ & $41 \pm 2^{\mathrm{c}}$ & $37 \pm 2$ & $39 \pm 2$ & $64 \pm 3$ & $70 \pm 4^{c}$ & $65 \pm 2$ & $67 \pm 3$ \\
\hline $20: 5, n-3$ & $6 \pm 0$ & $6 \pm 0$ & $6 \pm 1$ & $6 \pm 1$ & $9 \pm 1$ & $9 \pm 1$ & $9 \pm 1$ & $9 \pm 1$ & $24 \pm 2$ & $23 \pm 2$ & $22 \pm 2$ & $22 \pm 2$ \\
\hline $22: 4, n-6$ & $1 \pm 0$ & $1 \pm 0$ & $1 \pm 0$ & $1 \pm 0$ & $0 \pm 0$ & $0 \pm 0$ & $0 \pm 0$ & $0 \pm 0$ & $3 \pm 0$ & $3 \pm 0$ & $3 \pm 0$ & $3 \pm 0$ \\
\hline $22: 4, n-3$ & $5 \pm 1$ & $6 \pm 1$ & $4 \pm 0$ & $5 \pm 1$ & $0 \pm 0$ & $0 \pm 0$ & $0 \pm 0$ & $0 \pm 0$ & $33 \pm 3$ & $33 \pm 3$ & $29 \pm 2$ & $28 \pm 0$ \\
\hline $22: 6, n-3$ & $12 \pm 1$ & $12 \pm 1$ & $12 \pm 2$ & $11 \pm 2$ & $8 \pm 1$ & $8 \pm 1$ & $6 \pm 1$ & $6 \pm 1$ & $38 \pm 2$ & $41 \pm 3$ & $41 \pm 3$ & $41 \pm 2$ \\
\hline Total, mg/l & $434 \pm 39$ & $495 \pm 83$ & $499 \pm 75$ & $541 \pm 68$ & $809 \pm 38$ & $847 \pm 46$ & $864 \pm 50$ & $872 \pm 40$ & $621 \pm 59$ & $602 \pm 59$ & $639 \pm 24$ & $673 \pm 30$ \\
\hline
\end{tabular}

Mean \pm SEM ${ }^{a}$ The sum of identified fatty acids was indicated by 1000 . Difference between the groups: ${ }^{b} p<0.05$ or less. Significant effect of treatment: ${ }^{c} p<0.05$ or less 


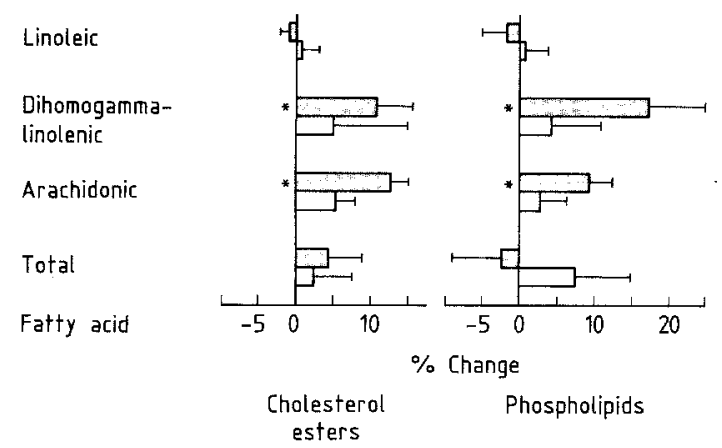

Fig. 1. Percentage changes in fatty acid contents in serum lipids by continuous subcutaneous insulin infusion ( $\boldsymbol{\square}$ ) and conventional insulin therapy $(\square)$. Mean \pm SEM of the changes. Significance of the changes: ${ }^{p} p<0.05$ or less

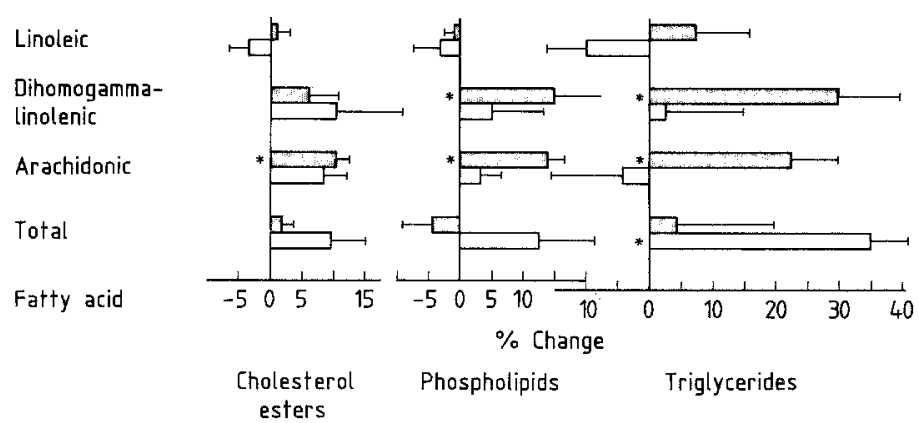

Fig. 2. Percentage change of fatty acid contents in serum lipids by fluctuation of diabetic control. $\mathbf{\square}=$ improved control $\left(\mathrm{HbA}_{1} \downarrow\right.$, $n=16) ; \square=$ impaired control $\left(\mathrm{HbA}_{1} \uparrow=, n=8\right)$. Mean $\pm \mathrm{SEM}$ of the changes. ${ }^{*} p<0.05$ or less

Table 3. Fatty acid compositions of erythrocytes and thrombocytes in Type 1 diabetic patients before and after treatment with continuous subcutaneous insulin infusion (CSII) and conventional injection therapy (CIT)

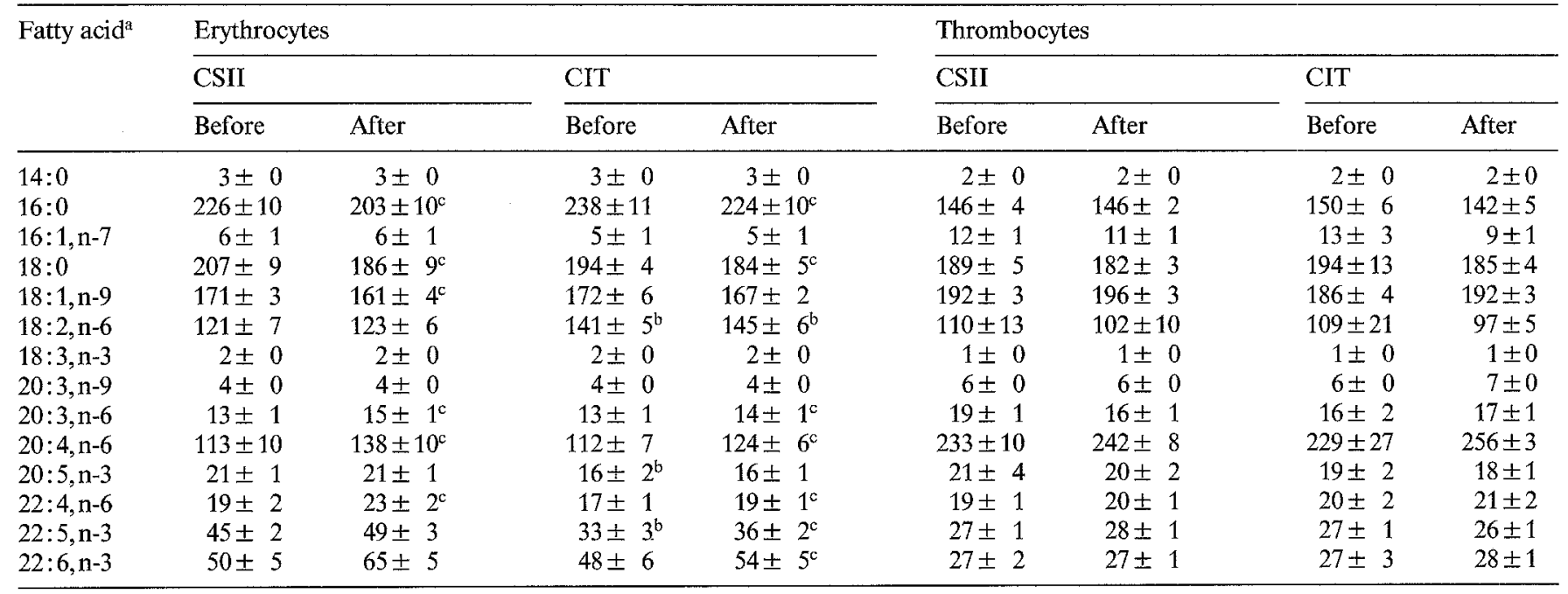

Mean \pm SEM ${ }^{a}$ The sum of identified fatty acids was indicated by 1000 . Significance between the groups: ${ }^{b} p<0.05$ or less. Effect of treatment: ${ }^{c} p<0.05$ or less

\section{Serum fatty acids}

In the serum lipids the intensified insulin treatment by the pump increased only the contents of n-6 PUFA and had no consistent effect on other fatty acids including linoleic acid (Table 2). Thus, the contents of DHGLA and arachidonic acid rose by up to $25 \%$ in serum triglycerides, cholesterol esters and phospholipids of the CSII group, whereas no significant changes were observed in the CIT group (Fig. 1).

The changes of n-6 PUFA were analyzed further according to improvement of diabetic control by the treatments using the fluctuation of glycosylated $\mathrm{HbA}_{1}$ as the indicator of a change in the control. In the patients in whom $\mathrm{HBA}_{1}$ fell the contents of arachidonic acid significantly rose $(p<0.05)$ in serum cholesterol esters, phospholipids and triglycerides, whereas no significant changes were found in patients with an unaltered or increased $\mathrm{HbA}_{1}$ level (Fig. 2). In serum phospholipids and triglycerides similar significant results were recorded for the DHGLA $(p<0.05)$, but no significant changes were found in linoleic acid of serum lipids. Poor diabetic control was associated with a significant increase in serum triglycerides $(p<0.01)$ and non-significant in cholesterol esters and phospholipids. Owing to the fluctuations of serum lipids neither significant differences nor changes were found in the absolute serum concentrations of individual fatty acids among the groups.

\section{Erythrocytes}

In contrast to the serum lipids, n-6 PUFA rose significantly $(p<0.05)$ in the erythrocytes of both treatment groups (Table 3 ). However, in the patients in whom $\mathrm{HbA}_{1}$ was decreased the $n-6$ PUFA/LA ratio was increased, whereas no consistent changes were found in other patients (Fig. 3). Furthermore, the changes in $\mathrm{HbA}_{1}$ were negatively correlated with those of $n-6 \mathrm{PU}-$ FA, e.g. arachidonic acid (Fig. 4), but not with that of linoleic acid. 


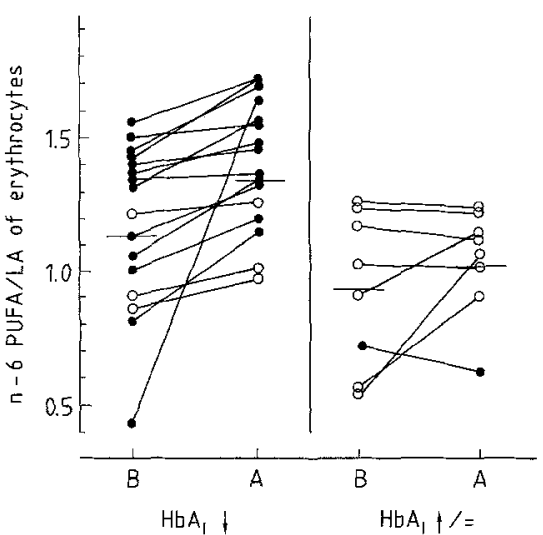

Fig.3. Ratios of $n-6$ PUFA to linoleic acid or erythrocytes before (B) and after (A) treatment with continuous subcutaneous insulin infusion $(\bullet)$ and conventional insulin regimen $(O)$. Means indicated by horizontal lines. $\mathrm{HbA}_{1} \downarrow=$ Patients in whom treatment decreased $\mathrm{HbA}_{1}, n=16 ; \mathrm{HbA}_{1} \uparrow=$ Patients with unchanged or increased $\mathrm{HbA}_{1}$ during treatment, $n=8$

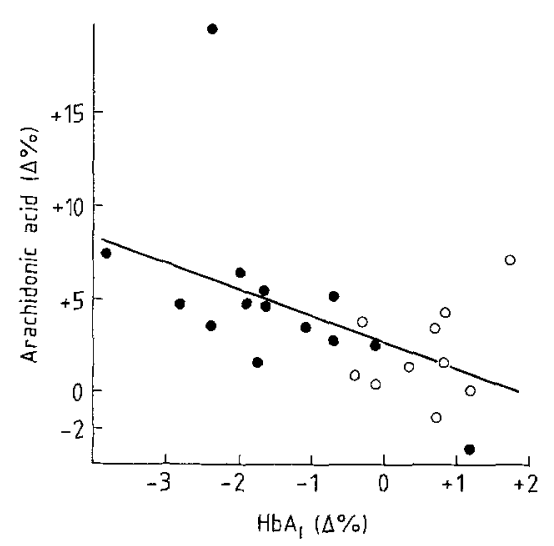

Fig. 4. Correlation of changes in $\mathrm{HbA}_{1}$ with those in percentage content of arachidonic acid or erythrocytes. $\bullet=$ patients treated with continuous subcutaneous insulin infusion, $O=$ conventional insulin regimen. $r=-0.455, p<0.05$

\section{Platelets}

The platelet fatty acid composition was not affected by CSII or CIT (Table 3).

\section{Discussion}

Our earlier findings [3] suggested that the low n-6 PUFA content of serum lipids and cellular membranes in women with Type 1 diabetes, especially in those with poor diabetic control, was due to impaired conversion of linoleic acid to the longer chain n-6 PUFA, i.e. DHGLA, arachidonic acid and docosatetraenoic acid. If this were true, improved diabetic control should be associated with an increase in n-6 PUFA. The present findings actually demonstrate that the intensified insulin treatment which improved diabetic control was associated with an increased contribution of n-6 PUFA of serum lipids and erythrocytes. This increase was pro- portionate to the improvement of diabetic control as indicated by the negative correlations between the changes in the fatty acid contents and that in the $\mathrm{HbA}_{1}$ level of erythrocytes.

Serum levels of n-6 PUFA are influenced by dietary intake of essential fatty acids, mainly linoleic acid and gamma-linolenic acid (GLNA; $18: 3, n-6$ ), chain-elongation and desaturation of linoleic acid to other n-6 PUFA, and incorporation of n-6 PUFA into tissue membrane lipids. The similarity of the initial values of serum lipids and their fatty acids suggests that the diets of the two treatment groups had been similar. The linoleic acid content in serum lipids and in the diet was not changed detectably during the treatment period. Dietary intake of arachidonic acid and other long chain n-6 PUFA is low and probably does not vary remarkably $[25,26]$. Thus, increased serum $\mathbf{n}-6$ PUFA are most likely formed endogenously from linoleic acid, even though the decreased utilization of n-6 PUFA to eicosanoids or conversion to peroxides during improved diabetic control can not be excluded. Some evidence has been presented that CSII might normalize the elevated thromboxane production in Type 1 diabetes [27]. The increase of n-6 PUFA in the erythrocytes of the present study and the unaltered platelet fatty acid composition suggest that the rise of serum n-6 PUFA, e.g. arachidonic acid, was not caused by a diminished incorporation of serum PUFA to membrane lipids during the improvement of the diabetic state. Thus, the increase of metabolic products of LA, n-6 PUFA, and the n-6 PUFA/LA ratios in serum lipids and erythrocytes concomitantly with the proportionate decrease of $\mathrm{HbA}_{1}$ suggest that the chain-elongation and desaturation of linoleic acid had been activated in patients with improved diabetic control.

The initial and rate-controlling step in the conversion of linoleic acid to arachidonic acid is the formation of $\gamma$-linolenic acid (GLNA $18: 3, \mathrm{n}-6$ ) by the $\Delta 6$ desaturase enzyme [4-10]. GLNA is further converted to DHGLA by chain elongation and further by the $\Delta 5 \mathrm{de}-$ saturase to arachidonic acid. The activities of these enzymes are decreased in experimental insulin deficiency [4-10]. In the present study the contents of DHGLA and arachidonic acid were slightly increased in serum lipids and especially in erythrocytes of the diabetic patients proportionately to the decrease in glycosylated haemoglobin.

No significant changes were observed in the contents of serum or erythrocyte eicosapentaenoic acid $(20: 5, n-3)$ and docosahexaenoic acid (22:6, n-3), even though the sum of $n-3$ PUFA tended to increase more in CSII than CIT. The n-3 PUFA are proposed to be the $\Delta 5$ and $\Delta 4$ desaturation and chain-elongation products of $\alpha$-linolenic acid (LNA 18:3, n-3). Our findings suggest that either the $\Delta 5$ and $\Delta 4$ desaturases and the chain elongation were not significantly activated by improved diabetic control or that only a negligible amount of tissue n-3 PUFA is derived from the dietary linoleic acid. 
The low n-3 PUFA in Type 1 diabetes [3] may be due to low dietary intake of these fatty acids or their enhanced consumption. On the other hand, if the $\Delta 4$ and $\Delta 5$ desaturases are not activated by improved diabetic balance, the rises of n-6 PUFA in serum lipids and erythrocytes could be secondary to the activation $\Delta 6$ desaturation and subsequently increased formation of GLNA and DHGLNA. Furthermore, no changes were observed in the concentrations of saturated and monoenoic fatty acids or their ratios, even though the activity of $\Delta 9$ desaturase, responsible for formation of monoenoic fatty acids, is decreased in the experimental insulin deficiency [8]. It is quite possible that changes greater than found in the diabetic control of our study could result in alterations of saturated/monoenoic fatty acid ratios and of n-3 PUFA.

The present experiments confirm the earlier results, suggesting the dependence of fatty acid composition of serum and erythrocyte membranes on the diabetic state in man [1-3], and imply that the insulin therapy enhances the conversion of linoleic acid to prostanoid precursor fatty acids. Further studies are needed to elucidate the degree to which alterations in prostanoid metabolism of diabetic patients [12-20] are based on disturbances in the synthesis of the precursor PUFA.

Acknowledgements. Ms A. Salolainen, P. Hoffström and M. Aarnio are acknowledged for technical and secretarial help. This study was supported by Nordisk Insulin Laboratories, Gentofte, Denmark and by a grant from the Finnish State Council for Medical Research.

\section{References}

1. Schrade W, Boehle E, Biegler R, Harmuth E (1963) Fatty acid composition of lipid fractions in diabetic serum. Lancet 1: 285-290

2. Tuna N, Frankhauser S, Goetz FC (1968) Total serum fatty acids in diabetes: relative and absolute concentrations of individual fatty acids. Am J Med Sci 255: 120-131

3. Tilvis RS, Miettinen TA (1985) Fatty acid composition of serum lipids, red cells and platelets in insulin-dependent diabetic women. J Clin Endocrinol Metab 61: 741-745

4. Mercuri O, Peluffo RO, Brenner RR (1966) Depression of microsomal desaturation of linoleic acid to gammalinolenic acid in the alloxan-diabetic rat. Biochim Biophys Acta 116: 409-411

5. MercuriO, Peluffo RO, Brenner RR.(1967) Effect of insulin on the oxidative desaturation of alfa-linolenic, oleic and palmitic acids. Lipids 2: 284-285

6. Peluffo RO, Ayala S, Brenner RR (1970) Metabolism of fatty acids of the linoleic acid series in testicles of diabetic rats. Am J Physiol 218: 669-673

7. Eck MG, Wynn JO, Carter WJ, Faas FH (1979) Fatty acid desaturation in experimental diabetes mellitus. Diabetes $28: 479-485$

8. Faas H, CarterWJ (1980) Altered fatty acid desaturation and microsomal fatty acid composition in the streptozotocin diabetic rat. Lipids 15: 953-961
9. Brenner RR, Peluffo RO, MercuriO, Restelli MA (1968) Effect of arachidonic acid in the alloxan-diabetic rat. Am J Physiol 215: 63-70

10. Holman RT, Johnson DM, GerrardJM, MauerSM, KupchoSandbergS, Brow DM (1983) Arachidonic acid deficiency in streptozotocin-induced diabetes. Proc Natl Acad Sci USA 80: 2375-2379

11. Moncada S, Vane JR (1979) Pharmacology and endogenous roles of prostaglandins, endoperoxides, thromboxane $\mathrm{A}_{2}$ and prostacyclin. Pharmacol Rev 30: 293-315

12. Bern MM (1978) Platelet functions in diabetes mellitus. Diabetes $27: 342-350$

13. Ganda OMP (1980) Pathogenesis of macrovascular disease in the human diabetes. Diabetes 29:931-942

14. Davis TME, Mitchell MD, Turner RC (1979) Prostacyclin and thromboxane metabolites in diabetes. Lancet 2: 789

15. Coldwell JA, Winocour PD, Halushka PV (1983) Do platelets have anything to do with diabetic microvascular disease? Diabetes 32 : 14-19

16. Waitzman MB (1979) Proposed metabolic dysfunction in diabetic microthromboses and microangiopathy. Metabolism 28: 401-406

17. Kalofoukes A, Lekasis I (1981) Changes of platelet phospholipids in diabetes mellitus. Diabetologia 21: 540-544

18. Morita J, Takahashi $R$, Ito H, Orimo H, Murota S (1983) Increased arachidonic acid content in platelet phospholipids from diabetic patients. Prostaglandin Leuk Med 11:33-41

19. Nordøy A, RodsetGM (1970 Platelet phospholipids and their functions in patients with juvenile diabetes and maturity onset diabetes. Diabetes 19: 698-702

20. Jones DB, Carter RD, Haites B, Mann SJ (1983) Low phospholipid arachidonic acid values in diabetic platelets. $\mathrm{Br}$ Med J286: $173-175$

21. Röschlau P, BerntE, GruberW (1974) Enzymatische Bestimmung des Gesamt-Cholesterins in Serum. Z Klin Chem Klin Biochem 12: 403-406

22. Wahlefeld AW (1974) Triglyceride determination after enzymatic hydrolysis. In: Bergmayer HV (ed) Methods in enzymatic analysis, Vol V. Academic Press, New York, p 1831

23. Welch SG, Boucher BJ (1978) A rapid micro-scale method for the measurement of haemoglobin $A_{1}$. Diabetologia 14: 209-211

24. Groop L, Maukonen L, Alopaeus K, Ylinen K, Teramo K, Pelkonen R (1982) The influence of rapid changes in blood glucose on glucosylated hemoglobin measured by microcolumn and macrocolumn chromatography. Ann Clin Res 14: 160-164

25. Nordøy A (1981) Lipids and trombogenesis. Ann Clin Res 13: $50-61$

26. Sanders TAB (1983) Dietary fat and platelet function. Clin Sci 65: 343-350

27. McDonaldJWD, DupreJ, Rodger NW, Champion MC, Webb CD, Ali M (1982) Comparison of platelet thromboxane synthesis in diabetic patients on conventional insulin therapy and continuous insulin infusions. Thromb Res 28:705

Received: 12 February 1986

and in revised form: 13 August 1986

Professor Tatu A. Miettinen, MD

Second Department of Medicine

University of Helsinki

SF-00290 Helsinki

Finland 